\title{
Neoantigen-based EpiCVAX vaccine initiates antitumor immunity in colorectal cancer
}

\author{
Victoria M. Kim,, ${ }^{1,2,3}$ Xingyi Pan,, ${ }^{1,2}$ Kevin C. Soares,, ${ }^{1,3}$ Nilofer S. Azad, ${ }^{1,2}$ Nita Ahuja, ${ }^{1,3}$ \\ Christopher J. Gamper, ${ }^{1,2}$ Alex B. Blair, ${ }^{1,2,3}$ Stephen Muth, ${ }^{1,2}$ Ding Ding, ${ }^{1,2}$ Brian H. Ladle, ${ }^{1,2}$ \\ and Lei Zheng ${ }^{1,2,3}$ \\ 'The Sidney Kimmel Comprehensive Cancer Center, ${ }^{2}$ Department of Oncology, and ${ }^{3}$ Department of Surgery, Johns Hopkins \\ University School of Medicine, Baltimore, Maryland, USA.
}

\begin{abstract}
Metastatic colorectal cancer (CRC) is poorly immunogenic, with limited neoantigens that can be targeted by cancer vaccine. Previous approaches to upregulate neoantigen have had limited success. In this study, we investigated the role of a DNA methyltransferase inhibitor (DNMTi), 5-aza-2'-deoxycytidine (DAC), in inducing cancer testis antigen (CTA) expression and evaluated the antitumor efficacy of a combinatorial approach with an epigenetically regulated cancer vaccine EpiCVAX and DAC. A murine model of metastatic CRC treated with combination therapy with an irradiated whole-cell CRC vaccine (GVAX) and DAC was used to assess the antitumor efficacy. DAC significantly induced expression of CTAs in CRC, including a new CTA Tra-P1A with a known neoepitope, P1A. Epigenetically modified EpiGVAX with DAC improved survival outcomes of GVAX. Using the epigenetically regulated antigen Tra-P1A as an example, our study suggests that the improved efficacy of EpiCVAX with DAC may due in part to the enhanced antigen-specific antitumor immune responses. This study shows that epigenetic therapy with DNMTi can not only induce new CTA expression but may also sensitize tumor cells for immunotherapy. Neoantigen-based EpiCVAX combined with DAC can improve the antitumor efficacy of GVAX by inducing antigen-specific antitumor $T$ cell responses to epigenetically regulated proteins.
\end{abstract}

Authorship note: VMK, XP, and KCS are co-first authors.

Conflict of interest: LZ receives grant support from Bristol-Meyer Squibb, Merck, iTeos, Amgen, NovaRock, InxMed, and Halozyme and received the royalty for licensing GVAX to Aduro BioTech. LZ is a paid consultant for/ advisory board member for Biosion, Alphamab, NovaRock, Akrevia, Sound Biologics, Fusun Biopharmaceutical, Foundation Medicine, DataRevive, and Mingruzhiyao. LZ holds shares at Alphamab and Mingruzhiyao. NA receives grant support from Cepheid and Astex and has served as consultant to Ethicon. NA has licensed methylation biomarkers to Cepheid.

Copyright: (c) 2020, American Society for Clinical Investigation.

Submitted: January 10, 2020

Accepted: April 8, 2020

Published: May 7, 2020.

Reference information: JCl Insight. 2020;5(9):e136368.

https://doi.org/10.1172/jci.

insight.136368.

\section{Introduction}

Colorectal cancer (CRC) is the third most commonly diagnosed cancer worldwide and the second leading cause of cancer-related death in the US. The 5-year survival rate for CRC combined at all stages is $65 \%$; however, about $25 \%$ of patients present with hepatic metastases at diagnosis and more than $70 \%$ patients will eventually develop hepatic metastases (1-5). Resection of colorectal liver metastases represents the only chance of cure; however, $75 \%$ of patients present with unresectable disease (3). For patients with CRC with unresectable liver metastases, chemotherapy alone only has a palliative role and rarely results in prolonged survival, with only a 11\% 5-year survival rate or less $(5,6)$. Immunotherapy, specifically PD-1/ PD-L1 checkpoint inhibitors, has provided significant advancements in treating more than 10 types of cancers (7). Indeed, checkpoint blockade in microsatellite-unstable metastatic CRC, which affects $15 \%$ of all patients with CRC, has shown significant clinical benefit (8). However, the vast majority of patients with CRC have microsatellite-stable disease, where checkpoint inhibitors are largely ineffective (9). The resistance of CRC to immunotherapy has in part been attributed to its poor tumor immunogenicity, with limited immune cell infiltration within the tumor microenvironment (TME) $(10,11)$.

Used in multiple tumor types, cancer vaccines induce infiltration of immune cells into the TME. A cancer vaccine composed of irradiated, allogeneic cancer cells and GM-CSF-secreting bystander cells, GVAX has been widely studied (12-14). In a phase Ib clinical trial, our group previously demonstrated that a single dose of neoadjuvant GVAX, composed of pancreatic cancer cells with or without immunomodulatory doses of cyclophosphamide, induced formation of tertiary lymphoid structures within 2 weeks of administration in $85 \%$ of vaccinated patients, whereas intratumoral tertiary lymphoid structures were not present in unvaccinated patients (ClinicalTrials.gov NCT007272441) $(15,16)$. These tertiary lymphoid structures contain organized and enriched $\mathrm{T}$ cell and $\mathrm{B}$ cell zones, and immunotherapy-naive patients who develop 
similar lymphoid structures have been shown to have improved survival $(16,17)$. Additionally, our group has shown that GVAX induces a systemic antigen-specific (mesothelin) T cell response $(15,18-22)$. After observing promising immunologic responses in pancreatic cancer patients receiving GVAX, the efficacy of GVAX in treating metastatic CRC was also tested. In a phase I study, 9 metastatic patients with CRC were given 4 doses of GVAX composed of CRC cells in the adjuvant setting; GVAX has been shown to be safe and enhance the production of antibodies against a specific tumor-associated antigen, MUC1, suggesting antigen-specific immune responses may be initiated by GVAX. Tumor-associated antigen immune responses were detected in 4 of 9 subjects (23). This immune response needs to be further studied; whether it is a humoral immune response or a $\mathrm{T}$ cell-mediated immune response initiated by cancer vaccine, these findings suggest that GVAX may initiate an antigen-specific immune response. However, GVAX only demonstrated limited clinical efficacy in various cancer types in clinical trials, despite stimulating immune response in patients (15), highlighting the needs to make tumor vaccination more potent.

Recent studies show that inhibition of epigenetic modification, specifically DNA methylation, can induce immune responses in tumor cells (24-26). DNA methylation inhibits expression of genes in promoter regions (27). An FDA-approved DNA methyltransferase inhibitor (DNMTi), 5-aza-2'-deoxycytidine (DAC), upregulates expression of cancer testis antigens (CTAs) in CRC cells, such as DAZL, which are normally expressed in early embryonic and germ cells but silenced in mature somatic cells due to DNA methylation at promoter regions $(25,28)$. Additionally, the CTA $M A G E$ family is subject to DNA methylation at promoter regions, and inhibition of DNA methylation allows the reexpression of MAGEs (29-31). Thus, inhibition of DNA methylation allows the reexpression of CTAs, which can potentially be recognized by the host immune system to enhance antitumor responses. DNMTi holds great potential for improving the efficacy of immunotherapy, including the cancer cell vaccine, GVAX. We propose that combinatorial GVAX and DNMTi can improve the survival of metastatic CRC by enhancing antigen-specific antitumor $\mathrm{CD}^{+} \mathrm{T}$ cell immune responses that target CTAs.

\section{Results}

Epigenetic modifier, DAC, induces CTA expression in a murine colorectal tumor CT26 cell line. A previous study has shown that a DNMTi, DAC, can upregulate CTA expression in multiple cancer cells, including CRCs, which can potentially be targeted by GVAX vaccine (25). To confirm their results and test the expression of more CTAs, we first investigated if DAC can induce the upregulation of a panel of 10 CTAs, selected from previously reported novel CTAs, based on literature (25). We conducted real-time PCR to study the expression of these genes before and after DAC treatment in the CT26 colorectal tumor cell line. Among 10 genes, 7 genes showed induction after DAC treatment, among which CTA Magea 9 showed a more than 40-fold induction and Oasl1 showed an approximately 13-fold induction at mRNA level (Figure 1A). One new CTA, Tra-P1A was also shown to be induced by DAC in the CT26 tumor line with a known epitope, P1A (LPYLGWLVF) (32). We observed a 35-fold induction of Tra-P1A expression at mRNA transcript level in CT26 tumor cells after DAC treatment (Figure 1B). Currently there is no antibody available to test Tra-P1A protein expression. As a result, we conclude that DAC can upregulate a wide range of CTA genes and could potentially prime tumor cells for more effective GVAX therapy.

Optimized GVAX vaccine using DAC (EpiGVAX) in combination with DAC improves survival outcomes of GVAX in a metastatic CRC murine tumor model. A previous study shows that CTAs are immunogenic and can initiate immune responses (33). Since we have demonstrated that DAC can induce CTA expression, we hypothesized these upregulated CTAs by DAC can potentially bolster the effectiveness of the cancer vaccine GVAX. As a result, combining DAC and GVAX may further improve survival outcomes of metastatic CRCs compared with GVAX alone. To test this hypothesis, we used a previously reported preclinical murine model of hepatic metastases (34), in which the CT26 colorectal tumor cells were injected into a hemispleen on day 0 to generate liver metastases, with removal of the injected hemispleen at the time of the operation/tumor inoculation. We first showed that combination of GVAX with DAC did not enhance the antitumor efficacy of GVAX (Supplemental Figure 1A and Supplemental Table 1; supplemental material available online with this article; https:// doi.org/10.1172/jci.insight.136368DS1). This result suggested that timing of DAC may be important in priming the TME for enhanced antitumor immune response. We then tested 3 dosing schedules for combination of GVAX and DAC as follows: DAC on days 3-7 before GVAX on day 11 (GVAX+DAC), DAC on days 11-15 with GVAX on day 11 (GVAX with DAC), and DAC on days 17-21 after GVAX treatment on day 11 (GVAX $\rightarrow$ DAC) (Supplemental Figure 1B). Mice treated with GVAX alone and GVAX+DAC regimens showed a markedly 
A

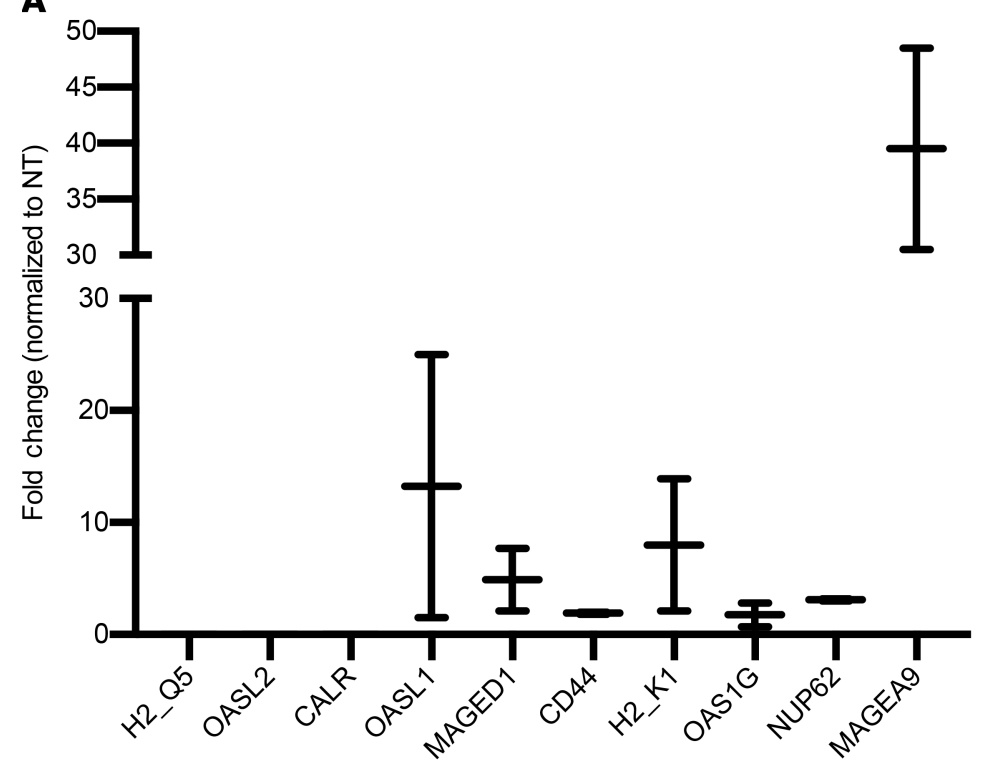

B

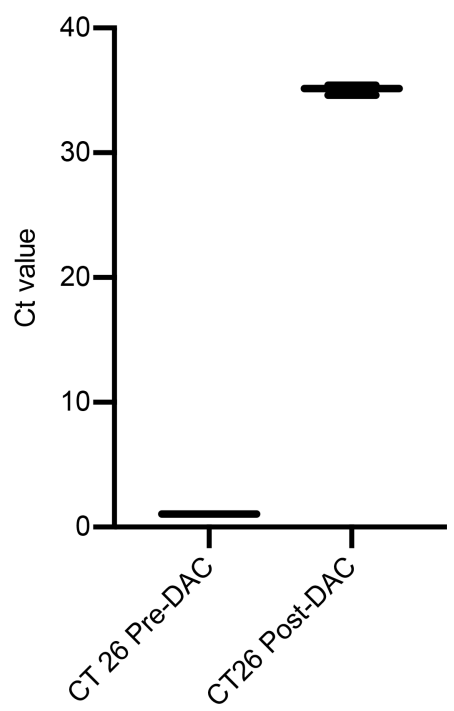

Figure 1. Decitabine induces cancer testis antigen expression in CT26 colorectal tumor cells. (A) CT26 tumor cells were cultured in a T75 flask and treated with decitabine (DAC) at a concentration of $1 \mu \mathrm{M}$ for 72 hours. Cells were harvested for quantitative real-time reverse transcription qPCR analysis, and expression of 10 CTAs at mRNA transcript level was tested. Data represent mean \pm SEM from triplicate of 1 representative experiment that was repeated twice. (B) Expression of 1 CTA Tra-P1A at mRNA transcript level was tested after DAC treatment. Both fold change compared with normal CT26 control and Ct values are presented. Data represent mean \pm SEM from triplicate of 1 representative experiment that was repeated twice.

enhanced antitumor response, with no visible evidence of tumor compared with mice without any treatment or with DAC as single agent (Supplemental Table 2). GVAX+DAC and GVAX with DAC regimens initiated a higher level of IFN- $\gamma$ expression in liver-infiltrating lymphocytes and splenocytes compared with GVAX $\rightarrow$ DAC (Supplemental Figure 1, C and D). Based on these results, we adopted a GVAX+DAC regimen for optimization. We hypothesized that the reason different combination regimens of GVAX with DAC did not further enhance the antitumor effect of GVAX was because without priming by DAC during the preparation of GVAX, GVAX was not able to initiate CTA-specific T cells that can match to the tumor antigens presented by tumor cells upregulated by DAC. To further improve the efficacy of combination therapy of GVAX with DAC, we generated a previously unknown vaccine EpiGVAX by pretreating the CT26 tumor cells used in the GVAX with DAC at a concentration of $1 \mu \mathrm{M}$ for 72 hours. Our hypothesis was that EpiGVAX is made of cancer cells that are pretreated with DAC, which have upregulated expression of a range of CTAs. Tumor cells within mice are treated with DAC systemically to upregulate CTA expression. We hypothesized T cells that are activated by EpiGVAX will recognize the tumor cells that present the same tumor antigens epigenetically regulated by DAC and, as a result, kill the tumor cells (see below). We tested the efficacy of EpiGVAX using the same hemispleen model (as above). We first found that EpiGVAX alone had a similar antitumor efficacy as GVAX alone and a slightly improved efficacy compared with no treatment; however, the EpiGVAX combined with DAC (EpiGVAX+DAC) regimen had a better efficacy than EpiGVAX alone (data not shown). This result confirmed our hypothesis that EpiGVAX itself may not be sufficient and that inclusion of DAC in vivo is crucial to augment the antitumor immune response and the overall efficacy of EpiGVAX. We subsequently repeated the experiment testing the efficacy of EpiGVAX+DAC, GVAX+DAC, and DAC alone (Figure 2A). Our analysis was preplanned to follow mice for survival for 50 days. Combination treatment of EpiGVAX+DAC showed the best antitumor response with statistically significant improved survival compared with single agent $\operatorname{GVAX}(P=0.0301)$. There was a survival advantage trend toward EpiGVAX+DAC over the GVAX+DAC regimen, but it was not statistically significant $(P=0.0671)$ (Figure $2 \mathrm{~B}$ and Table 1). After 50 days, mice given the EpiGVAX+DAC regimen started to die at a similar rate as mice given GVAX+DAC or GVAX regimen, and half of them died by 90 days, which is likely due to insufficient treatment, with only 1 treatment cycle. These results show that the EpiGVAX+DAC regimen improves survival outcomes of GVAX in a metastatic CRC murine tumor model.

EpiGVAX in combination with DAC enhances antigen-specific antitumor immune responses in a metastatic CRC murine tumor model. DAC can induce upregulation of Tra-P1A in CT26 tumor cells; therefore, the 

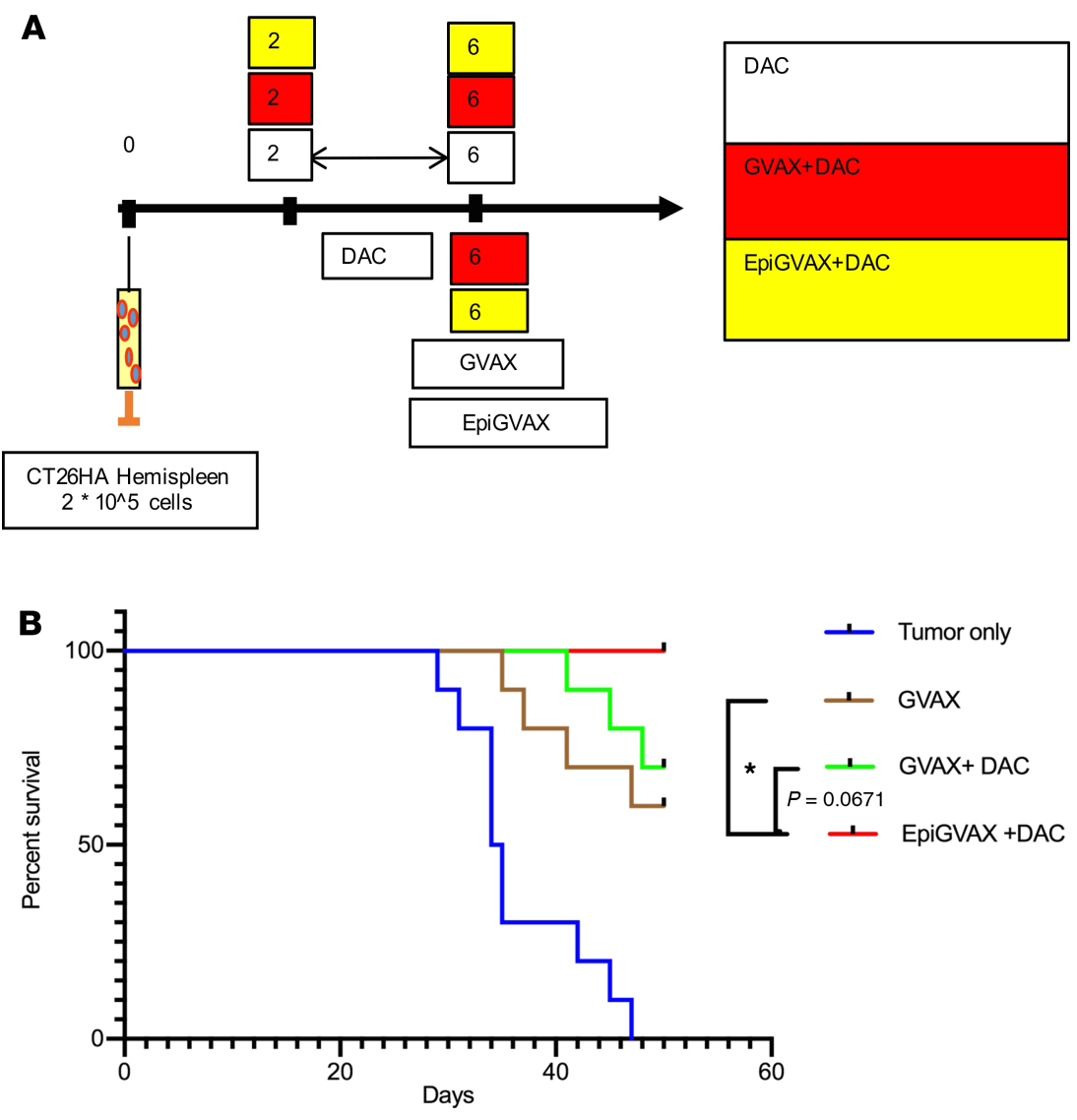

Figure 2. The EpiGVAX+DAC treatment regimen improves the survival outcome of GVAX in a murine liver metastatic model. (A) $2 \times 10^{5}$ CT26 tumor cells were inoculated via a hemispleen surgery on day 0 . Mice were treated with GVAX or EpiCVAX at day 6, and DAC was dosed from day 2 to day 6 consecutively for 5 days. Mice were followed for survival. (B) Mice were followed for survival for 50 days, and the survival percentage at day 50 was calculated. $n=10$ mice per group. Data represent 1 representative experiment that was repeated twice. Survival statistical analysis was done by using log-rank test adjusted by multiple testing. ${ }^{*} P<0.05$.

P1A epitope can potentially be used as neoepitope to demonstrate an antigen-specific antitumor $\mathrm{T}$ cell response (Figure 1B). The P1A epitope binds with MHC class I molecule that can be recognized by CD $8^{+}$ $\mathrm{T}$ cells. A previous study has also shown that antitumor systemic immunity of GVAX is $\mathrm{CD} 8^{+}$dependent (13). We sought to use P1A to interrogate whether EpiGVAX could induce an antigen-specific CD8 T cell response. To test levels of P1A-specific antitumor $\mathrm{T}$ cell responses induced by different treatment groups, flow cytometry analysis was performed using P1A-specific pentamer staining on $\mathrm{CD} 8^{+} \mathrm{T}$ cells isolated from murine liver-infiltrating lymphocytes. The P1A-specific $\mathrm{CD} 8^{+} \mathrm{T}$ cell population expanded significantly upon GVAX vaccine treatment compared with control mice without tumor $(P=0.0017)$. EpiGVAX+DAC showed a trend toward further increasing the $\mathrm{P} 1 \mathrm{~A}^{+} \mathrm{CD} 8^{+} \mathrm{T}$ cell population; however, this increase was not statistically significant $(P=0.4374)$. Interestingly, a lower percentage of the $\mathrm{P}^{+} \mathrm{A}^{+} \mathrm{CD} 8^{+} \mathrm{T}$ cell population was observed in the GVAX+DAC treatment group compared with the group treated with GVAX alone $(P=0.0896)$. A decreased percentage of $\mathrm{P} \mathrm{A}^{+} \mathrm{CD} 8^{+} \mathrm{T}$ cell population was also observed in the DAC alone group compared with the tumor-bearing control mice without treatment, although the difference was not statistically significant $(P=0.6052)$ (Figure 3$)$. Using CTA Tra-P1A as an example, this study suggests that both GVAX alone and EpiGVAX+DAC were able to induce the expansion of Tra-P1A antigen-specific $\mathrm{CD} 8^{+} \mathrm{T}$ cell pools, which may account for their observed survival benefits.

\section{Discussion}

Our study confirmed that DNMTi was able to upregulate the expression of CTAs, and, more importantly, we identified many CTAs that can also be epigenetically regulated by DNMTi, including Oasl1, Oas2, Magea9, and Tra-P1A. This result suggests DNMTi may initiate reexpression of a wide array of CTAs. With the rationale 
Table 1. Day 50 interim analysis of mouse survival

\begin{tabular}{ccc}
\hline Treatment groups $(\boldsymbol{n}=\mathbf{1 0})$ & \% Survival at day $\mathbf{5 0}$ & $\begin{array}{c}\text { P value } \\
\text { (versus EpiGVAX+DAC) } \\
\text { by 2-sided } \chi^{2} \text { test }\end{array}$ \\
Tumor only & 0 & $<0.0001$ \\
GVAX & 60 & $<0.05$ \\
GVAX+DAC & 70 & 0.0603 \\
\hline
\end{tabular}

that DNMTi may sensitize tumor cells for immunotherapy through induced CTA expression, our study developed a previously known epigenetically modified cancer vaccine approach and demonstrated improved efficacy with combination therapy with EpiGVAX+DAC in a preclinical model compared with GVAX or DAC as a single agent. EpiGVAX+DAC is able to initiate an antitumor-specific $\mathrm{CD} 8^{+} \mathrm{T}$ cell response by expanding the neoantigen-specific $\mathrm{CD} 8^{+} \mathrm{T}$ cell pool, which may account for their yielded survival benefits.

In this study, EpiGVAX is made of cancer cells that are pretreated with DAC, which have upregulated expression of a range of CTAs. GM-CSF cytokine recruits dendritic cells to process and present CTAs from EpiGVAX to activate T cells. Tumor cells within mice are treated with DAC systemically to upregulate CTA expression. T cells that are activated by EpiGVAX will recognize the tumor cells that present the same tumor antigens epigenetically regulated by DAC and as a result kill the tumor cells. Without DAC treatment, EpiGVAX-induced CTA-specific T cells would not match to the tumor antigens presented by tumor cells (Figure 4). The study results confirmed our hypothesis and suggested that the combination treatment with EpiGVAX+DAC showed improved antitumor efficacy and potential neoantigen $\mathrm{T}$ cell expansion in vivo. They also explained why a simple regimen of GVAX+DAC did not show a survival benefit, as without priming by DAC, GVAX was not able to initiate CTA-specific T cells that can match to the tumor antigens presented by tumor cells upregulated by DAC. However, the survival advantage of the GVAX+DAC regimen over that of GVAX was not statistically significant. The $\mathrm{T}$ cell expansion property of the GVAX+DAC regimen over that of GVAX was also not statistically significant. These results suggest that upregulation of CTA expression may not be sufficient to sensitize the tumor cells for immunotherapy, thus only yielding limited efficacy and neoantigen-specific $\mathrm{T}$ cell expansion. Interestingly, a lower percentage of P1A antigen-specific $C D 8^{+} \mathrm{T}$ cells was observed in the GVAX+DAC regimen compared with GVAX alone, as a similar decreasing trend was also observed in the DAC single-agent regimen. This result suggests a potential role of DAC in decreasing neoantigen-specific $\mathrm{T}$ cell response, which may due to its toxicity to $\mathrm{T}$ cells, and requires further studies. This may also explain the decreased efficacy of GVAX when combined with DAC in postvaccination conditions, as cytotoxic T cells may be killed by DAC. In addition, the immunomodulating roles of DAC to GVAX will be further studied.

This study supports the rationale that DNMTi can induce neoantigen-specific $\mathrm{T}$ cell response by inducing the expression of CTAs that can be recognized as neoantigens and yield survival benefit. However, the survival advantage of combination treatment of EpiGVAX+DAC is still limited, which suggests that upregulation of neoantigens itself may not be sufficient to initiate neoantigen-specific $\mathrm{T}$ cell responses. Other crucial mechanisms in generating neoantigen-specific $\mathrm{T}$ cell responses remain to be explored. In addition, the neoantigen-specific $\mathrm{T}$ cells may present exhausted phenotype within TME. Thus, additional immunomodulators may also be necessary to prevent the $\mathrm{CD} 8^{+}$cytotoxic $\mathrm{T}$ cells from undergoing immunoexhaustion, such as PD-1/PD-L1 checkpoint blockade. To address the concern of $\mathrm{T}$ cell exhaustion, the efficacy of combination therapy with an EpiGVAX+DAC+anti-PD1 regimen will be tested.

One limitation of this study is that only a single colon cell line is studied. CT26 is the only well-characterized murine syngeneic colon carcinoma cell line. Therefore, the conclusion in this preclinical study will need to be validated in the human clinical studies of GVAX. To initiate liver metastasis we used the hemispleen model, which does not fully mimic the progression of liver metastasis in CRC, which is colorectum to liver metastasis. The colorectal microenvironment may alter the epigenetic states of CRC, and thus orthotopic transplantation to the colon will be conducted to further validate our findings. Based on this study, the sequence of treatment with DAC and GVAX appears to be critical. Having DNMTi treatment before GVAX treatment yielded the better survival benefit. However, in human studies, such a combination 


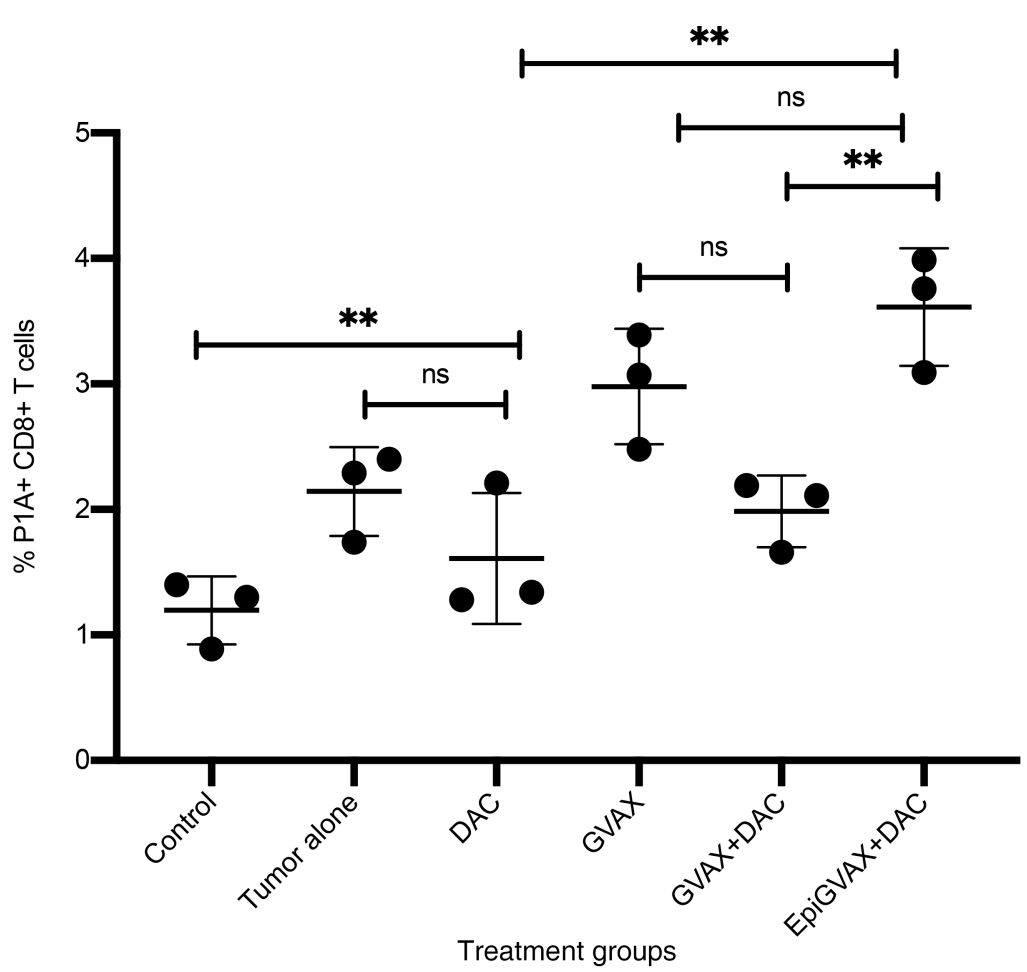

Figure 3. EpiGVAX+DAC induces Tra-P1A antigen-specific antitumor immune response. Liver metastatic CT26 colorectal tumor-bearing mice were sacrificed on day 17 after surgery, and flow cytometry analysis was performed on CD8 ${ }^{+} T$ cells isolated from liver-infiltrating immune cells isolated from mice. $n=3$ mice per group. Percentages of P1A+CD8 ${ }^{+} T$ cells were compared among different treatment groups. Isolated immune cells from mice in the same treatment group were pooled and measured in triplicate. Data represent 1 representative experiment that was repeated twice. Multiple comparisons of unpaired 1-way ANOVA test with Tukey's $P$ value adjustment were used for statistical analysis. ${ }^{*} P<0.01$.

of DNMTi and EpiGVAX will be administered as multiple cycles; as a result, the differential effect of the sequencing of DNMTi in inducing CTA expression for priming GVAX treatment may be abrogated. Thus, the effects of multiple cycles of therapy will be investigated in future studies.

As previously discussed, DAC may have a cytotoxic effect on $\mathrm{T}$ cells, requiring further research to confirm the optimal dosage in human studies. Potential effects of DAC on other immune cells, including myeloid cells, remain to be explored. Tra-P1A is specific for mice with no human homolog; moreover, neoantigen expression in humans is highly dynamic and patient specific (35). Further studies are required to identify consistent neoantigens as biomarkers to track neoantigen-specific $\mathrm{T}$ cell responses initiated by combination therapy with EpiGVAX+DAC in human studies.

Our study shows that the epigenetic modifier DNMTi can not only induce new CTA expression, but also sensitize tumor cells for immunotherapy and yield an improved efficacy with combination therapy of EpiGVAX+DAC in a preclinical model by inducing neoantigen-specific $\mathrm{T}$ cell response. This study uncovers a role of epigenetic modifiers in modulating immune responses by regulation of CTA expression. As a result, different epigenetic modifiers, including DNMTi, may have broader applications in inducing CTA expression that can be combined with cancer cell vaccines to augment antigen-specific $\mathrm{T}$ cell activation and increase the efficacy of cancer cell vaccines in different tumor types, particularly solid tumors with fewer tumor-infiltrating lymphocytes and less $\mathrm{T}$ cell activation. Regarding clinical studies, in a phase I clinical trial, 9 patients with unresectable liver-predominant metastases were given DAC through hepatic arterial infusion on 5 consecutive days every 4 weeks. The results showed that DAC has a safe profile at a dose level of $20 \mathrm{mg} / \mathrm{m}^{2} / \mathrm{d}$, with grades 1 and 2 hematological toxicity being the most frequent treatment-related adverse event; none of the patients experienced treatment-limiting adverse events. More importantly, upregulation of 21 of the 30 CTAs being tested was observed comparing pretreatment and 


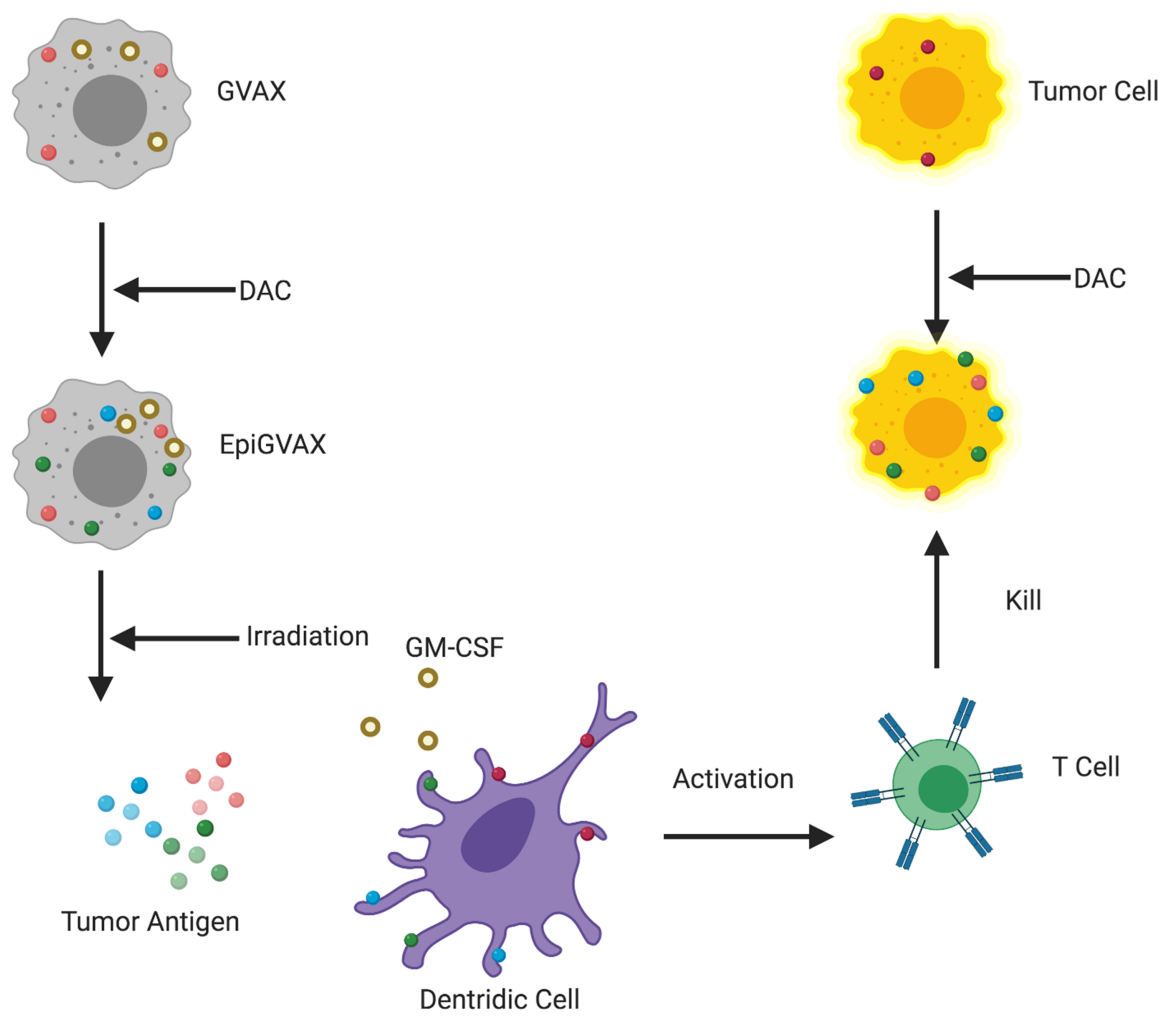

Figure 4. A working model of EpiGVAX in treating colorectal cancer. GVAX is made of cancer cells that express tumor antigens and are genetically modified to express GM-CSF cytokine. EpiCVAX is made of cancer cells that are pretreated with DAC, which have upregulated expression of a range of CTAs. CM-CSF cytokine recruits dendritic cells to process and present CTAs from EpiCVAX to activate T cells. Tumor cells within mice are treated with DAC systemically to upregulate CTA expression. T cells that are activated by EpiGVAX will recognize the tumor cells that present the same tumor antigens epigenetically regulated by DAC, and as a result these T cells kill the tumor cells.

posttreatment biopsies (36). In another phase I clinical trial in which a small number of patients with advanced NSCLC who progressed after receiving low-dose epigenetic therapy DAC were given immune checkpoint therapy, about $20 \%$ of the patients responded to immune checkpoint therapy alone $(37,38)$. These clinical trial results further support our hypothesis that DAC can be used safely to sensitize immunotherapy through upregulation of CTA expression. Due to the highly dynamic nature of neoantigens present in patients, further studies are required to identify consistent neoantigens as biomarkers to track neoantigen-specific T cell responses following combination therapy with EpiGVAX and DAC. Nevertheless, combination therapy with EpiGVAX+DAC showed improved antitumor efficacy in a CRC preclinical model, which warrants further investigation in human studies for CRCs.

\section{Methods}

Cell lines and medium. CT26 BALB/c colorectal carcinoma was generated by injection of methylcholanthrene as previously described (39). The CT26-HA cell line was established by transfecting CT26 cells with a plasmid encoding the influenza-derived HA gene by electroporation (40). CT26 and CT26-HA cells were maintained in an incubator at $37^{\circ} \mathrm{C}$ with $5 \% \mathrm{CO}_{2}$ in RMPI 1640 media (Life Technologies) supplemented with 10\% heat-inactivated fetal bovine serum (HI-FBS, Benchmark), 1\% penicillin/ streptomycin (pen/strep, Life Technologies), 1\% Minimum Essential Media-Non-Essential Amino Acids (MEM-NEAA, Life Technologies), 1\% sodium pyruvate (MilliporeSigma), 1\% L-glutamine (Life Technologies), 1\% HEPES (Life Technologies), and 0.05\% 2-mercaptoethanol (MilliporeSigma). $\mathrm{B} 78 \mathrm{H} 1$ is an MHC class $\mathrm{I}^{-}$murine fibroblast cell line engineered to secrete GM-CSF (41). B78H1 cells were cultured in an incubator at $37^{\circ} \mathrm{C}$ with $5 \% \mathrm{CO}_{2}$ in RMPI 1640 media (Life Technologies) supplemented with 10\% HI-FBS, $1 \%$ pen/strep, and 1\% L-glutamine. Harvested tumor-infiltrating immune cells were processed in complete media, which is made of RPMI media supplemented with 
10\% HI-FBS, 1\% pen/strep, 1\% HEPES, 1\% MEM-NEAA, 1\% L-glutamine, and 0.05\% 2-mercaptoethanol. The cell lines were tested for mycoplasma every 6 months.

Mice and in vivo experiments. Six- to eight-week-old BALB/c female mice were purchased from The Jackson Laboratory. For survival studies, mice were monitored for up to 100 days. Mice considered to have reached a "survival endpoint," including hunched posture, lethargy, dehydration, and rough hair coat, were euthanized.

CT26 tumor cell inoculation was performed when the mice reached 7-9 weeks of age. The hemispleen injection was used for tumor inoculation on day 0 as described previously (41). In brief, mice were first anesthetized, a left subcostal incision was made, and the spleen was then eviscerated, clipped, and divided into 2 half spleens. $2 \times 10^{5}$ CT26 or $2 \times 10^{5}$ CT26-HA tumor cells resuspended in $100 \mu \mathrm{L}$ PBS (Gibco) were injected into the spleen half without leakage and flushed with $150 \mu \mathrm{L}$ PBS in the same syringe. The half of the spleen through which the tumor cells were injected was then removed to avoid residual tumor cells, leaving the other half of the spleen in the mouse. The peritoneum and the skin were then sutured. Following this procedure, diffuse liver metastases developed, and all untreated mice died in 4-6 weeks $(34,42)$.

DAC (MilliporeSigma, A3656) was dissolved in DMSO and was administered i.p. at $0.14 \mathrm{mg} / \mathrm{kg}$ per mouse on followed the treatment schema shown in Figure 2A and Supplement Figure 1B. Murine GVAX vaccine was made by combining $1 \times 10^{6} \mathrm{CT} 26$ cells and $1 \times 10^{6} \mathrm{~B} 78 \mathrm{H} 1$ cells in PBS to make a total cell concentration of $20 \times 10^{6} \mathrm{cells} / \mathrm{mL}$. Murine EpiGVAX vaccine was made by combining $1 \times 10^{6} \mathrm{DAC}$-treated CT26 cells and $1 \times 10^{6} \mathrm{~B} 78 \mathrm{H} 1$ cells in PBS to make a total cell concentration of $20 \times 10^{6}$ cells $/ \mathrm{mL}$. CT26 cells were pretreated with DAC for 72 hours at a concentration of $1 \mu \mathrm{M}$ before making EpiGVAX. The cell suspension was then irradiated at $50 \mathrm{~Gy}$ and administered subcutaneously in 3 locations, bilateral flanks and one of the upper limbs, at $100 \mu \mathrm{L}$ per injection with a total number of $6 \times 10^{6}$ cells injected per mouse. Cyclophosphamide (Bristol-Myers Squibb) was administered i.p. at $100 \mathrm{mg} / \mathrm{kg} 1$ day before GVAX vaccination for Treg depletion as previously reported $(15,43)$.

DAC treatment. CT26 cells were cultured in $30 \mathrm{ml}$ media in a T175 cell flask (Corning). When CT26 cells reached $80 \%$ confluence, cells were treated with DAC at a concentration of $1 \mu \mathrm{M}$ for 72 hours, and then the cells were harvested for RNA analysis.

Quantitative real-time reverse transcription PCR. TRIzol Reagent (Thermo Fisher Scientific) was used to extract RNA from CT26 cells. RNA was then converted to cDNA using the Superscript III First Strand Synthesis Supermix Kit (Thermo Fisher Scientific). Quantitative RT-PCR was performed on the StepOnePlus Real-time PCR System (Thermo Fisher Scientific) and analyzed by the StepOne software (V2.1). The expression of genes was quantified by SYBR Green (Applied Biosystems) fluorescence. All gene expression was normalized to the expression of $\beta$-actin. All PCR reactions were performed in triplicate.

Processing of liver metastasis-infiltrating lymphocytes. Liver metastasis-infiltrating lymphocytes were analyzed on day 15 following CT26 tumor inoculation, with different treatment courses shown in Figure 3 . Each liver was mechanically processed sequentially through $100-\mu \mathrm{m}$ and $40-\mu \mathrm{m}$ nylon filters and brought to a volume of $25 \mathrm{~mL}$ complete media. Cell suspensions were centrifuged at $300 \mathrm{~g}$ for 5 minutes. Liver cells were resuspended in $4 \mathrm{~mL}$ ACK lysis buffer (Quality Biological) for 2 minutes and were subsequently centrifuged at $300 \mathrm{~g}$ for 5 minutes. Liver cell pellets were then resuspended in $6 \mathrm{~mL} \mathrm{80 \%} \mathrm{Percoll} \mathrm{(GE} \mathrm{Healthcare} \mathrm{Life} \mathrm{Sciences),} \mathrm{overlaid} \mathrm{with} 6 \mathrm{~mL} \mathrm{40 \%} \mathrm{Percoll,} \mathrm{and} \mathrm{centrifuged}$ for 25 minutes at $600 \mathrm{~g}$ with no brake at room temperature. The lymphocyte layer was collected by an 18 -gauge needle and resuspended in $10 \mathrm{~mL}$ complete media.

Pentamer staining and flow cytometry. Following the isolation of liver metastasis colorectal tumor-infiltrating immune cells, $\mathrm{CD} 8^{+} \mathrm{T}$ cell enrichment was performed using a CD8- isolation kit (Life Technologies) according to the protocols provided by the manufacturer. After enrichment, $\mathrm{CD} 8^{+} \mathrm{T}$ cells were stained with the Live Dead Aqua Dead Cell Kit (Invitrogen). The CD8 ${ }^{+} \mathrm{T}$ cells were washed and subsequently blocked with mouse Fc antibody (BD Pharmingen) for 10 minutes on ice followed by incubation of P1A Pentamer-PE (Proimmune, 150) at $10 \mu \mathrm{L} /$ per $100 \mu \mathrm{L}$ sample for 15 minutes at room temperature. Cells were washed with PBS and assayed on Cytoflex cytometer (Beckman).

Statistics. Kaplan-Meier curve was used for survival curve analysis, and log-rank test was used for survival curve comparison. One-way ANOVA was used for comparisons among multiple groups ( $\geq 3)$, and Tukey's $P$ value adjustment was used for multiple testing. Wilcoxon test or $t$ test was used continuous variable comparison between 2 unpaired groups, as appropriate. $\chi^{2}$ test or Fisher's exact test was used for categorical variable comparison between 2 unpaired groups, as appropriate. All tests were 2 tailed, and $P$ values of less than 0.05 was considered statistically significant. Statistical analyses were done using GraphPad Prism software. 
Study approval. All studies and maintenance of mice were conducted with the approval of and in accordance with the guidelines of the Johns Hopkins Institutional Animal Care and Use Committee.

\section{Author contributions}

LZ conceived the concept. VMK, XP, KCS, CJG, BHL, and LZ designed the research studies. VMK, XP, $\mathrm{KCS}, \mathrm{ABB}, \mathrm{SM}$, and BHL conducted experiments. VMK, XP, and KCS acquired data. VMK, XP, KCS, DD, CJG, NAS, NA, BHL, and LZ analyzed data. All authors critically reviewed the manuscript.

\section{Acknowledgments}

This work was supported by The Zhang Family Gift Fund (to LZ) and the Susie's Cause Colon Cancer Foundation grant (to LZ), NIH grants R01 CA169702 and R01 CA197296 (to LZ), National Cancer Institute Specialized Programs of Research Excellence in Gastrointestinal Cancers grant P50 CA062924 (to LZ), and Sidney Kimmel Comprehensive Cancer Center grant P30 CA006973 (to LZ). This work was supported by National Cancer Institute Cancer Center Support Grant P30 CA006973 (Cancer Center Core Grant) (to BHL). This work was supported by the National Heart, Lung, and Blood Institute (1K08HL108346-01) (to CJG). We would like to thank Montana O'Dell from Texas Tech University for providing assistance for this project.

Address correspondence to: Lei Zheng or Brian H. Ladle, 1650 Orleans Street, CRB1 Room 488 (LZ) or Room 205 (BHL), Baltimore, Maryland 21287, USA. Phone: 410.502.6241; Email: lzheng6@jhmi.edu (LZ). Phone: 443.287.3534; Email: bladle@jhmi.edu (BHL).

KCS's present address is: Department of Surgery, Hepatopancreatobiliary Service, Memorial Sloan Kettering Cancer Center, New York, New York, USA.

NA's present address is: Department of Surgery, Yale University, New Haven, Connecticut, USA.

1. House MG, et al. Comparison of adjuvant systemic chemotherapy with or without hepatic arterial infusional chemotherapy after hepatic resection for metastatic colorectal cancer. Ann Surg. 2011;254(6):851-856.

2. Jegatheeswaran S, Mason JM, Hancock HC, Siriwardena AK. The liver-first approach to the management of colorectal cancer with synchronous hepatic metastases: a systematic review. JAMA Surg. 2013;148(4):385-391.

3. Jemal A, Thomas A, Murray T, Thun M. Cancer statistics, 2002. CA Cancer J Clin. 2002;52(1):23-47.

4. Tomlinson JS, et al. Actual 10-year survival after resection of colorectal liver metastases defines cure. J Clin Oncol. 2007;25(29):4575-4580.

5. van de Velde CJH. Colorectal cancer: treatment of liver metastases of colorectal cancer. Ann Oncol. 2005;16(supp1_2):ii144-ii149.

6. Misiakos EP, Karidis NP, Kouraklis G. Current treatment for colorectal liver metastases. World J Gastroenterol. 2011;17(36):4067-4075.

7. Wu X, et al. Application of PD-1 blockade in cancer immunotherapy. Comput Struct Biotechnol J. 2019;17:661-674

8. Le DT, et al. Mismatch repair deficiency predicts response of solid tumors to PD-1 blockade. Science. 2017;357(6349):409-413

9. Kalyan A, Kircher S, Shah H, Mulcahy M, Benson A. Updates on immunotherapy for colorectal cancer. J Gastrointest Oncol. 2018;9(1):160-169.

10. Dienstmann R, Vermeulen L, Guinney J, Kopetz S, Tejpar S, Tabernero J. Consensus molecular subtypes and the evolution of precision medicine in colorectal cancer. Nat Rev Cancer. 2017;17(2):79-92.

11. Roelands J, et al. Immunogenomic classification of colorectal cancer and therapeutic implications. Int J Mol Sci. 2017;18(10):E2229.

12. Dranoff G. GM-CSF-based cancer vaccines. Immunol Rev. 2002;188:147-154.

13. Dranoff G, et al. Vaccination with irradiated tumor cells engineered to secrete murine granulocyte-macrophage colony-stimulating factor stimulates potent, specific, and long-lasting anti-tumor immunity. Proc Natl Acad Sci USA. 1993;90(8):3539-3543.

14. Dranoff G, et al. A phase I study of vaccination with autologous, irradiated melanoma cells engineered to secrete human granulocyte-macrophage colony stimulating factor. Hum Gene Ther. 1997;8(1):111-123.

15. Lutz E, et al. A lethally irradiated allogeneic granulocyte-macrophage colony stimulating factor-secreting tumor vaccine for pancreatic adenocarcinoma. A phase II trial of safety, efficacy, and immune activation. Ann Surg. 2011;253(2):328-335.

16. Lutz ER, et al. Immunotherapy converts nonimmunogenic pancreatic tumors into immunogenic foci of immune regulation. Cancer Immunol Res. 2014;2(7):616-631.

17. Dieu-Nosjean MC, et al. Long-term survival for patients with non-small-cell lung cancer with intratumoral lymphoid structures. J Clin Oncol. 2008;26(27):4410-4417.

18. Jaffee EM, et al. Novel allogeneic granulocyte-macrophage colony-stimulating factor-secreting tumor vaccine for pancreatic cancer: a phase I trial of safety and immune activation. J Clin Oncol. 2001;19(1):145-156.

19. Jaffee EM, et al. Development and characterization of a cytokine-secreting pancreatic adenocarcinoma vaccine from primary tumors for use in clinical trials. Cancer J Sci Am. 1998;4(3):194-203. 
20. Laheru D, et al. Allogeneic granulocyte macrophage colony-stimulating factor-secreting tumor immunotherapy alone or in sequence with cyclophosphamide for metastatic pancreatic cancer: a pilot study of safety, feasibility, and immune activation Clin Cancer Res. 2008;14(5):1455-1463.

21. Le DT, et al. Evaluation of ipilimumab in combination with allogeneic pancreatic tumor cells transfected with a GM-CSF gene in previously treated pancreatic cancer. J Immunother. 2013;36(7):382-389.

22. Thomas AM, et al. Mesothelin-specific CD8(+) T cell responses provide evidence of in vivo cross-priming by antigen-presenting cells in vaccinated pancreatic cancer patients. J Exp Med. 2004;200(3):297-306.

23. Zheng L, et al. A safety and feasibility study of an allogeneic colon cancer cell vaccine administered with a granulocytemacrophage colony stimulating factor-producing bystander cell line in patients with metastatic colorectal cancer. Ann Surg Oncol. 2014;21(12):3931-3937.

24. $\mathrm{Li} \mathrm{H}$, et al. Immune regulation by low doses of the DNA methyltransferase inhibitor 5-azacitidine in common human epithelial cancers. Oncotarget. 2014;5(3):587-598.

25. Siebenkäs C, et al. Inhibiting DNA methylation activates cancer testis antigens and expression of the antigen processing and presentation machinery in colon and ovarian cancer cells. PLoS One. 2017;12(6):e0179501.

26. Wrangle J, et al. Alterations of immune response of non-small cell lung cancer with azacytidine. Oncotarget. 2013;4(11):2067-2079.

27. Jin B, Li Y, Robertson KD. DNA methylation: superior or subordinate in the epigenetic hierarchy? Genes Cancer. 2011;2(6):607-617.

28. Chiappinelli KB, et al. Inhibiting DNA methylation causes an interferon response in cancer via dsRNA including endogenous retroviruses. Cell. 2015;162(5):974-986.

29. De Smet C, Loriot A, Boon T. Promoter-dependent mechanism leading to selective hypomethylation within the 5' region of gene MAGE-A1 in tumor cells. Mol Cell Biol. 2004;24(11):4781-4790.

30. De Smet C, Lurquin C, Lethé B, Martelange V, Boon T. DNA methylation is the primary silencing mechanism for a set of germ line- and tumor-specific genes with a CpG-rich promoter. Mol Cell Biol. 1999;19(11):7327-7335.

31. Karpf AR, Bai S, James SR, Mohler JL, Wilson EM. Increased expression of androgen receptor coregulator MAGE-11 in prostate cancer by DNA hypomethylation and cyclic AMP. Mol Cancer Res. 2009;7(4):523-535.

32. Mroz P, Vatansever F, Muchowicz A, Hamblin MR. Photodynamic therapy of murine mastocytoma induces specific immune responses against the cancer/testis antigen P1A. Cancer Res. 2013;73(21):6462-6470.

33. Kirkin AF, Dzhandzhugazyan KN, Zeuthen J. Cancer/testis antigens: structural and immunobiological properties. Cancer Invest. $2002 ; 20(2): 222-236$.

34. Soares KC, et al. A preclinical murine model of hepatic metastases. J Vis Exp. 2014;(91):51677.

35. Guo Y, Lei K, Tang L. Neoantigen vaccine delivery for personalized anticancer immunotherapy. Front Immunol. 2018;9:1499.

36. Jansen YJL, et al. Phase I clinical trial of decitabine (5-aza-2'-deoxycytidine) administered by hepatic arterial infusion in patients with unresectable liver-predominant metastases. ESMO Open. 2019;4(2):e000464.

37. Brahmer JR, et al. Safety and activity of anti-PD-L1 antibody in patients with advanced cancer. N Engl JMed. 2012;366(26):2455-2465.

38. Topalian SL, et al. Safety, activity, and immune correlates of anti-PD-1 antibody in cancer. N Engl J Med. 2012;366(26):2443-2454.

39. Corbett TH, Griswold DP, Roberts BJ, Peckham JC, Schabel FM. Tumor induction relationships in development of transplantable cancers of the colon in mice for chemotherapy assays, with a note on carcinogen structure. Cancer Res. 1975;35(9):2434-2439.

40. Scheffer SR, et al. Apoptotic, but not necrotic, tumor cell vaccines induce a potent immune response in vivo. Int J Cancer. 2003;103(2):205-211.

41. Jain A, Slansky JE, Matey LC, Allen HE, Pardoll DM, Schulick RD. Synergistic effect of a granulocyte-macrophage colony-stimulating factor-transduced tumor vaccine and systemic interleukin- 2 in the treatment of murine colorectal cancer hepatic metastases. Ann Surg Oncol. 2003;10(7):810-820.

42. Soares KC, et al. PD-1/PD-L1 blockade together with vaccine therapy facilitates effector T-cell infiltration into pancreatic tumors. J Immunother. 2015;38(1):1-11.

43. Machiels JP, et al. Cyclophosphamide, doxorubicin, and paclitaxel enhance the antitumor immune response of granulocyte/macrophage-colony stimulating factor-secreting whole-cell vaccines in HER-2/neu tolerized mice. Cancer Res. 2001;61(9):3689-3697. 